\title{
Shielding Requirements of a SPECT Insert for Installation in a PET/MRI System
}

\author{
Debora Salvado $^{1}$, Student Member, IEEE, Kjell Erlandsson ${ }^{1}$, and Brian F. Hutton ${ }^{1,2}$, Senior Member, IEEE
}

\begin{abstract}
The objective of this work is to evaluate the shielding requirements of a SPECT insert for installation in the Siemens Biograph mMR in order to perform simultaneous SPECT/MR imaging of the human brain. We intend to use the radionuclides ${ }^{99 \mathrm{~m}} \mathrm{Tc}$, ${ }^{123} \mathrm{I}$ and ${ }^{111} \mathrm{In}$. The main photopeaks of these radionuclides have the following energies: 140.5, 159.0, 171.3 and $245.4 \mathrm{keV}$. There is also about $\sim 3 \%$ of emission probability of high energy gamma photons for ${ }^{123} \mathrm{I}$ in the range of 248-784 $\mathrm{keV}$. The main constraints to the design of the gamma shielding are the presence of high energy photons, the weight, the MR compatibility and the PET LSO crystals intrinsic activity. We used GATE to simulate a SPECT acquisition, defining an MRI system with LSO crystals, a partial SPECT ring and a NEMA phantom. We also defined a lead $(\mathrm{Pb})$ base plate (BP) to simulate the support system and three $\mathrm{Pb}$ shielding volumes with variable thickness: front and end (FE), back (B), and lateral (L) shield. These volumes reduce interference from out-of-field activity, LSO intrinsic activity and edge effects, respectively. We performed 4 sets of simulations, with variable $F E$, variable $B$, variable $L$ and variable $B P$ thickness, respectively, with a NEMA phantom filled with $185 \mathrm{MBq}$ of ${ }^{123} \mathrm{I}$ or ${ }^{111} \mathrm{In}$. For all simulations, we compared the different energy spectra and countdistribution plots. Results show that a $\mathrm{Pb}$ shielding configuration with a thickness of $6 \mathrm{~mm}-\mathrm{F}, 2 \mathrm{~mm}-\mathrm{E}$, $3 \mathrm{~mm}-\mathrm{B}$, and $5 \mathrm{~mm}-\mathrm{L}$ is appropriate for the insert. For ${ }^{123}$ I there is still a high contribution from high energy photons, as the amount of shielding is limited by weight, however this contribution is likely to be overestimated in the simulations as compared to practice. The effect of the LSO intrinsic activity is negligible at the energies of interest.
\end{abstract}

Index Terms-SPECT/MR, SPECT insert, multimodality, gamma shielding.

\section{INTRODUCTION}

$\mathbf{T}$ HE main goal of this work was to evaluate the shielding requirements of a SPECT insert for installation in the Siemens Biograph mMR [1] using Monte Carlo-based simulations.

\footnotetext{
${ }^{1}$ Institute of Nuclear Medicine, University College London, UK.

${ }^{2}$ Centre for Medical Radiation Physics, University of Wollongong, Australia.

Debora Salvado is supported by a $\mathrm{PhD}$ Fellowship, reference SFRH/BD/88093/2012, by the Portuguese national funding agency for science, research and technology (FCT). This work was done as part of the INSERT collaboration, which is supported by the EC under the FP7-HEALTH program (305311). UCL/UCLH research is supported by the NIHR University College London Hospitals Biomedical Research Centre.
}

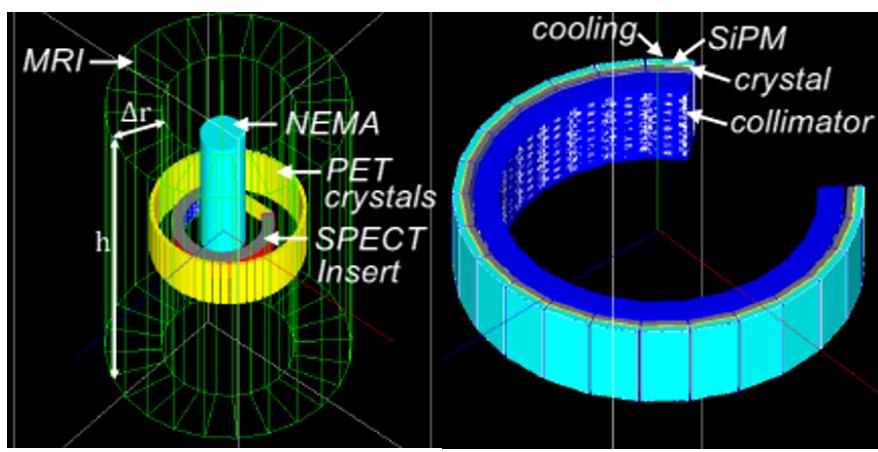

Fig. 1. Configuration of the simulation setup used in GATE.

The SPECT insert will consist of a partial ring of 20 detectors with $5 \times 10 \mathrm{~cm}$ SiPM readout units, $8 \mathrm{~mm}$ thickness CsI crystals and a mini-slit-slat (MSS) collimator [2]. The expected intrinsic resolution is $0.8 \mathrm{~mm}[3]$ and the target resolution $10 \mathrm{~mm}$ at the centre of the FOV. With the purpose of enhanced stratification of brain tumour patients, we intend to use ${ }^{99 \mathrm{~m}} \mathrm{Tc},{ }^{123} \mathrm{I}$ and ${ }^{111} \mathrm{In}$ as the radionuclides of interest when performing simultaneous SPECT/MR imaging. The main photopeaks of these radionuclides have the following energies: $140.5 \mathrm{keV}\left({ }^{99 \mathrm{~m}} \mathrm{Tc}\right), 159.0 \mathrm{keV}\left({ }^{123} \mathrm{I}\right)$, $171.3 \mathrm{keV}$ and $245.4 \mathrm{keV}\left({ }^{111} \mathrm{In}\right)$. In addition, there is a small percentage $(\sim 3 \%)$ of emission probability of high energy gamma photons for ${ }^{123} \mathrm{I}$ in the range of $248-784 \mathrm{keV}$.

Considering that the SPECT insert will be installed in a PET/MR system, there might be some backscatter in the bore, specially due to the high energy gamma photons. In addition, the PET LSO crystals have intrinsic activity [4] due to the presence of ${ }^{176} \mathrm{Lu}$, which emits photons at $88.4 \mathrm{keV}, 201.8 \mathrm{keV}$ and $306.8 \mathrm{keV}$ [5]. These photons can then be detected by the SPECT insert if appropriate shielding is not in place. Therefore our objectives were to assess and evaluate the need for gamma shielding of the detection units, like in a standard gamma camera, in order to minimise the backscatter due to activity in the patient outside the FOV and the LSO intrinsic activity interference.

\section{Materials And Methods}

We used GATE [6] to simulate the SPECT acquisition. Primarily, we defined three main volumes with specific attenuating media: the MRI system, the SPECT insert and a NEMA phantom (Fig. 1). The MRI system was set up as a cylindrical aluminium volume, with a thickness of $250 \mathrm{~mm}$ and a length of $1594 \mathrm{~mm}$, and 56 blocks of LSO in a ring 


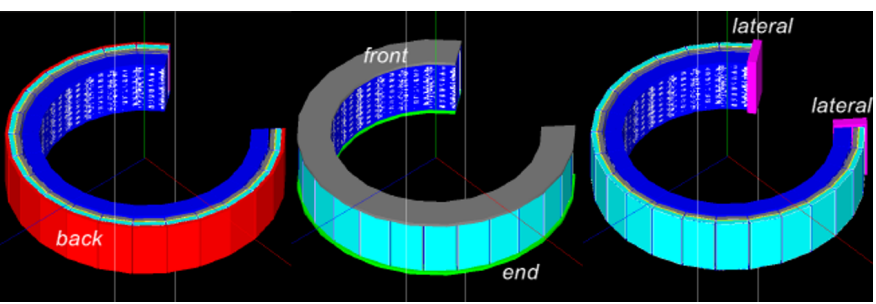

Fig. 2. Shielding volumes.

configuration to mimic the PET crystals ring. Each block had a size of $20 \times 32 \times 256 \mathrm{~mm}^{3}$ and a ${ }^{176} \mathrm{Lu}$-based activity of $45 \mathrm{kBq}$, amounting to a total intrinsic activity for the ring of $2.5 \mathrm{MBq}$. The SPECT insert was defined as a partial ring of 20 detection modules composed of a water cooling block, a silicon layer and a CsI crystal in a trapezoidal shape. For each detection module, a corresponding lead $(\mathrm{Pb})$ collimator was added with the MSS design. The NEMA phantom was defined as a water cylinder with a radius of $102 \mathrm{~mm}$ and a length of $700 \mathrm{~mm}$.

Secondly, we defined a $\mathrm{Pb}$ base plate $(\mathrm{BP})$ to simulate the support system and three $\mathrm{Pb}$ shielding volumes with variable thickness $t$ : front and end (FE), back (B), and lateral (L) shield (Fig. 2). FE shield reduces the interference of photons from out-of-field activity. B shield reduces interference from backscatter and LSO intrinsic activity. Finally, L shield reduces detector edge effects.

Finally we used the visualization software OGLSX to confirm the right positioning of the volumes in the GATE space.

We performed 4 sets of simulations with a ${ }^{123}$ I-filled NEMA phantom with $185 \mathrm{MBq}$ of activity:

- variable thickness $\mathrm{FE} t_{\mathrm{FE}}=\{0,5,6,10,15\} \mathrm{mm}$ and remaining shield volumes thickness fixed $t_{\mathrm{B}}=6 \mathrm{~mm}$, $t_{\mathrm{L}}=15 \mathrm{~mm}, t_{\mathrm{BP}}=3 \mathrm{~mm}$ (an extra simulation with different $\mathrm{F}$ and $\mathrm{E}$ shields thickness, $t_{\mathrm{F}}=6 \mathrm{~mm}$ and $t_{\mathrm{E}}=2 \mathrm{~mm}$, was also performed);

- variable thickness $\mathrm{B} t_{\mathrm{B}}=\{0,2,3,4,6\} \mathrm{mm}$ and remaining shield volumes thickness fixed $t_{\mathrm{FE}}=15 \mathrm{~mm}$, $t_{\mathrm{L}}=15 \mathrm{~mm}, t_{\mathrm{BP}}=3 \mathrm{~mm}$;

- variable thickness $\mathrm{L} t_{\mathrm{L}}=\{0,1,3,5,10,15\} \mathrm{mm}$ and remaining shield volumes thickness fixed $t_{\mathrm{FE}}=15 \mathrm{~mm}$, $t_{\mathrm{B}}=6 \mathrm{~mm}, t_{\mathrm{BP}}=3 \mathrm{~mm}$;

- variable thickness $\mathrm{BP} t_{\mathrm{BP}}=\{0,1,2,3\} \mathrm{mm}$ and remaining shield volumes thickness fixed $t_{\mathrm{FE}}=15 \mathrm{~mm}$, $t_{\mathrm{B}}=6 \mathrm{~mm}, t_{\mathrm{L}}=15 \mathrm{~mm}$.

The same set of simulations were also performed for a ${ }^{111}$ In-filled NEMA phantom.

All simulations were run for 1-second acquisition time. This is a short acquisition time when compared to practice, but it is enough to obtain the energy spectra and reduces the simulation time significantly. In the case of ${ }^{123} \mathrm{I}$, only two photopeaks were modelled to further simplify the simulation, $159 \mathrm{keV}(83 \%)$ and $529 \mathrm{keV}(3 \%)$ to account for the high energy photons.

The obtained energy spectra and detected counts distribution were extracted from the GATE .root files and

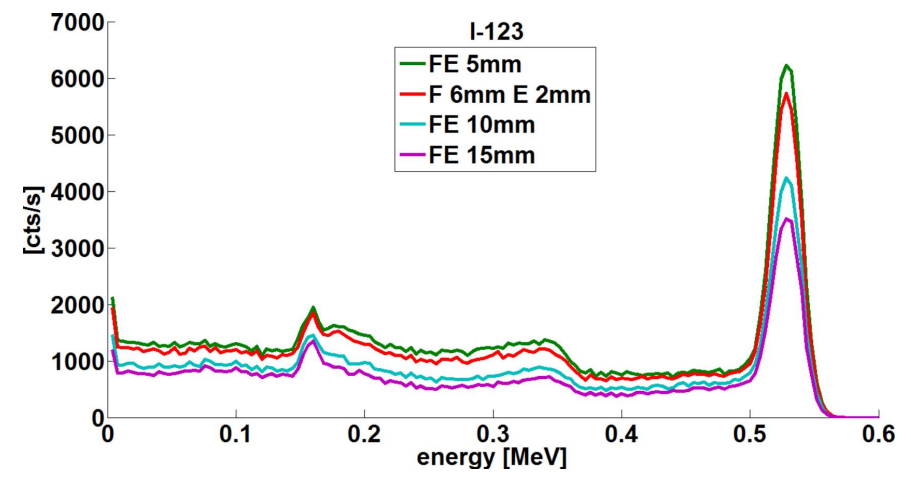

Fig. 3. Energy spectra obtained with ${ }^{123}$ I for variable FE shields.

compared for all cases, to select the most suited shielding configuration for the SPECT insert.

With the final design of shielding, the effects of the LSO intrinsic activity were assessed comparing non-shielded to shielded SPECT insert. Furthermore, a simulation with ${ }^{99 \mathrm{~m}} \mathrm{Tc}$ was also performed to obtain the energy spectrum for this radionuclide.

\section{RESULTS}

Fig. 3, 5 and 13 show the energy spectra obtained with ${ }^{123}$ I for simulations with variable FE, B and BP thickness, respectively. The different colours correspond to a different thickness of the shielding. For viewing purposes (different scale), the spectrum for simulations without shielding is not shown. For ${ }^{111}$ In, Fig. 4, 6 and 14 show the energy spectra for the simulations described previously.

Fig. 7, 9 and 11 show the planar, trans-axial and last detector count-distribution plots for ${ }^{123} \mathrm{I}$ simulations with variable FE, B and L thickness, respectively. Each dot corresponds to a detected event. Peak 1 refers to $159 \mathrm{keV}$ and peak 2 to $529 \mathrm{keV}$. For ${ }^{111} \mathrm{In}$, Fig. 8,10 and 12 show the corresponding count-distribution plots for the simulations described previously. In this case, peak 1 refers to $171 \mathrm{keV}$ and peak 2 to $245 \mathrm{keV}$.

Fig. 15 shows the energy spectra for simulations with ${ }^{176} \mathrm{Lu}$ with and without the final shielding design.

Fig. 16 shows the energy spectra obtained with ${ }^{99 \mathrm{~m}} \mathrm{Tc}$, ${ }^{123} \mathrm{I}$ and ${ }^{111} \mathrm{In}$ for the final shielding design. The ${ }^{99 \mathrm{~m}} \mathrm{Tc}$ and ${ }^{111}$ In photopeaks have good definition, but the ${ }^{123} \mathrm{I}$ one has a high background.

\section{Discussion}

Analysing the plots of Fig. 3-4, both energy spectra show a reduction of counts with thicker $\mathrm{FE}$ shielding. Considering the simulations with $t_{\mathrm{F}}=6 \mathrm{~mm}$ and $t_{\mathrm{E}}=2 \mathrm{~mm}$, the accumulation of counts at the front and back edge of the detectors is reduced for the first peak of ${ }^{123} \mathrm{I}$, but not for the second high energy peak, when compared to the no FE shielding case (Fig. 7). However, the shielding weight required to stop these photons would overload the system. For ${ }^{111}$ In (Fig. 8), the count-distribution along the detectors is uniform, as opposed to the absence of $\mathrm{FE}$ shielding case. 


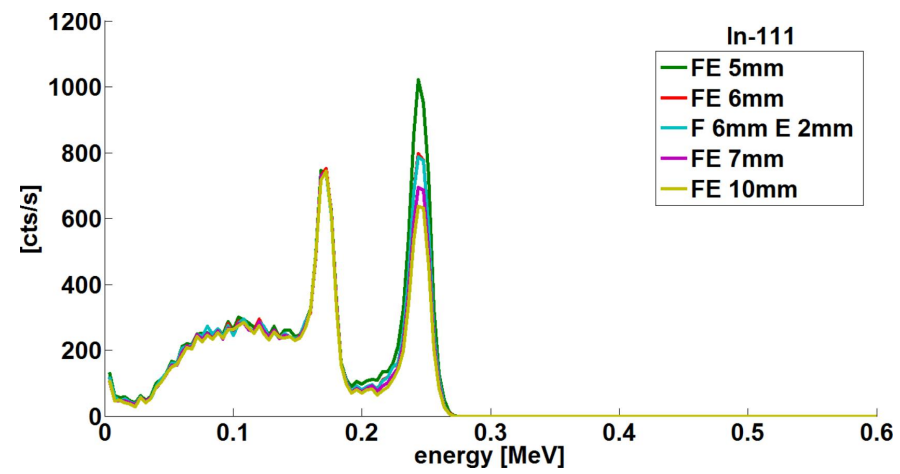

Fig. 4. Energy spectra obtained with ${ }^{111}$ In for variable FE shields.

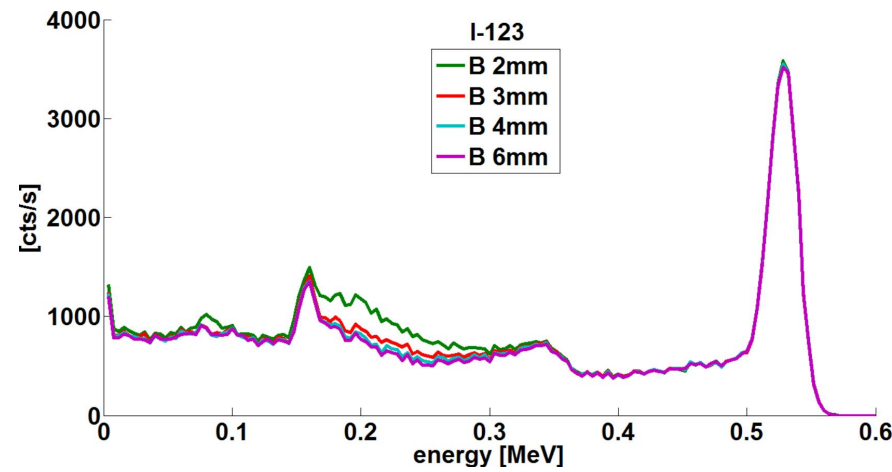

Fig. 5. Energy spectra obtained with ${ }^{123} \mathrm{I}$ for variable B shields.

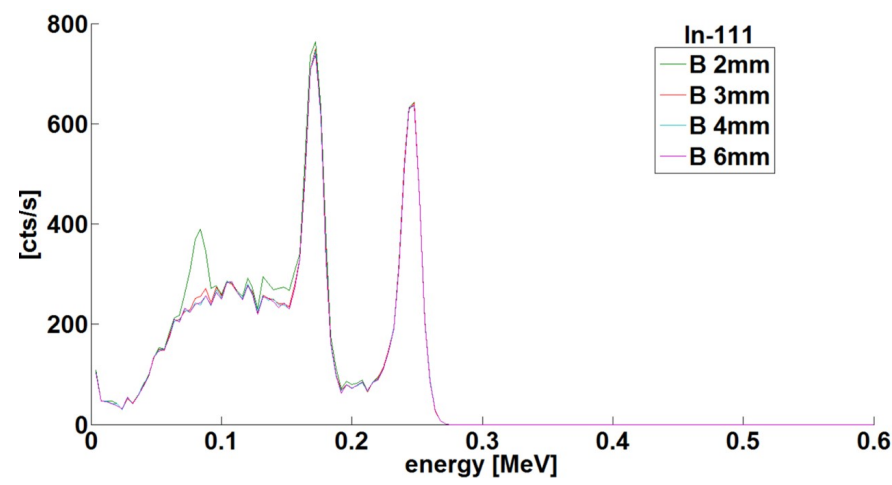

Fig. 6. Energy spectra obtained with ${ }^{111}$ In for variable B shields.
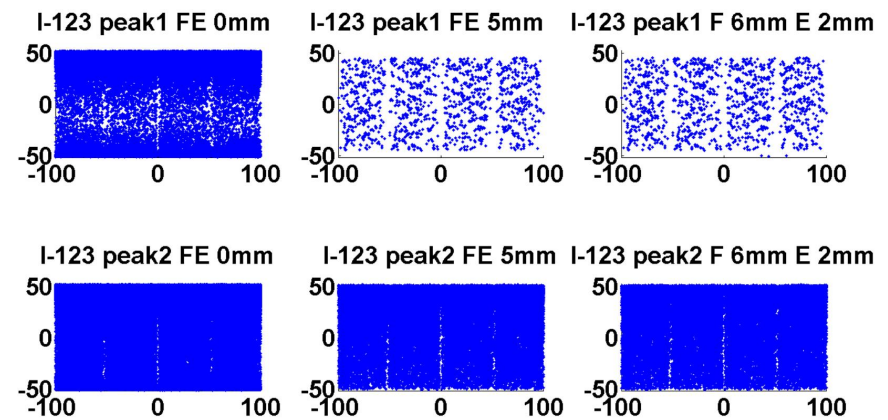

Fig. 7. Distribution of counts along four detectors in the ring obtained with ${ }^{123} \mathrm{I}$ for variable FE shields.
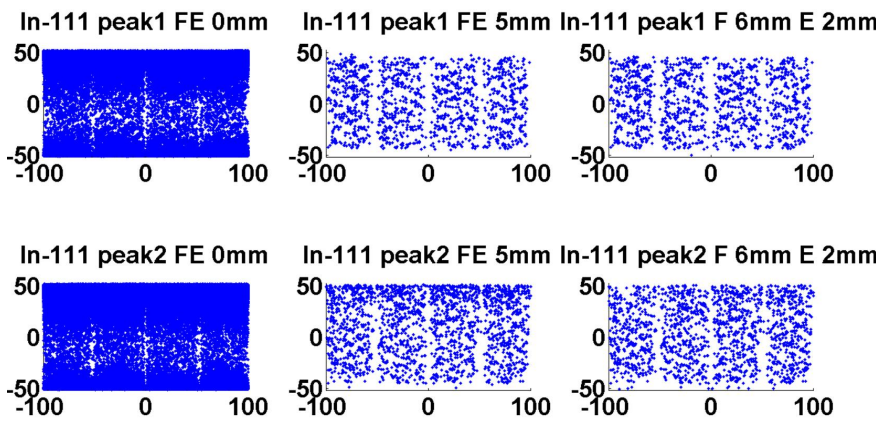

Fig. 8. Distribution of counts along four detectors in the ring obtained with ${ }^{111} \mathrm{In}$ for variable $\mathrm{FE}$ shields.

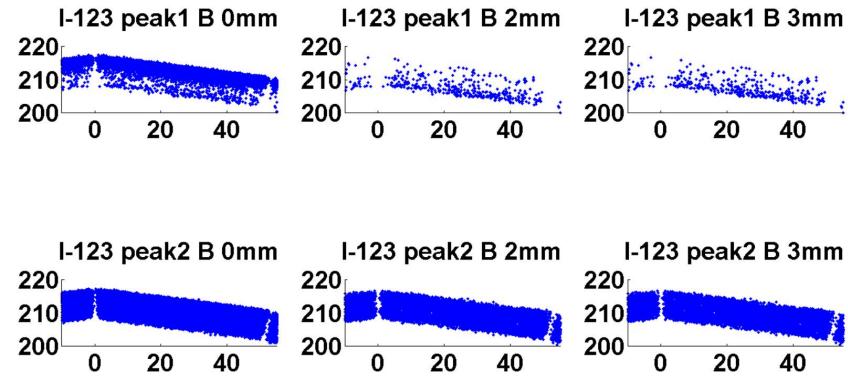

Fig. 9. Trans-axial distribution of counts along one detector in the ring obtained with ${ }^{123} \mathrm{I}$ for variable B shield.
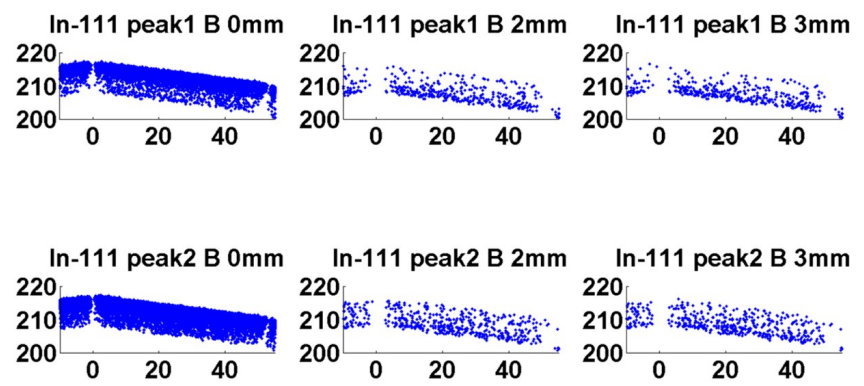

Fig. 10. Trans-axial distribution of counts along one detector in the ring obtained with ${ }^{111}$ In for variable B shield.
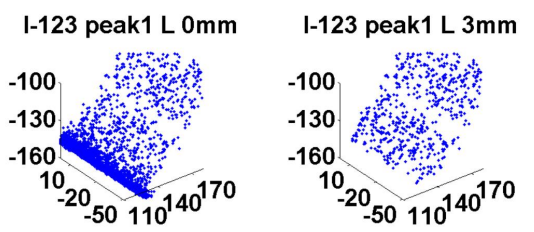

I-123 peak1 L 5mm
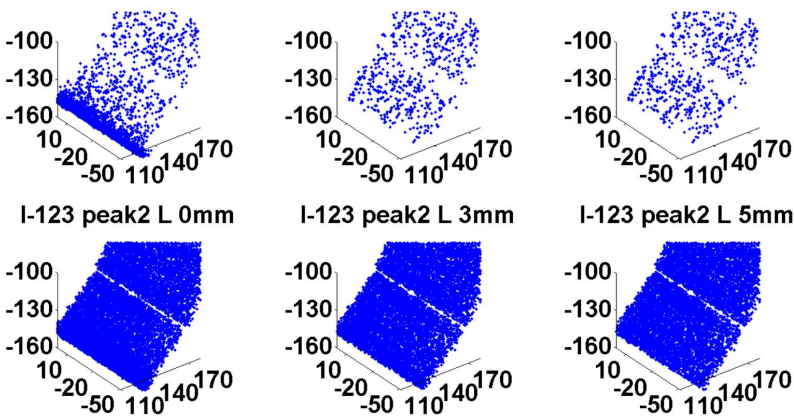

I-123 peak2 L $5 \mathrm{~mm}$

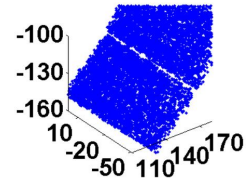

Fig. 11. Distribution of counts along the last detector of the partial ring obtained with ${ }^{123}$ I for variable $\mathrm{L}$ shield. 

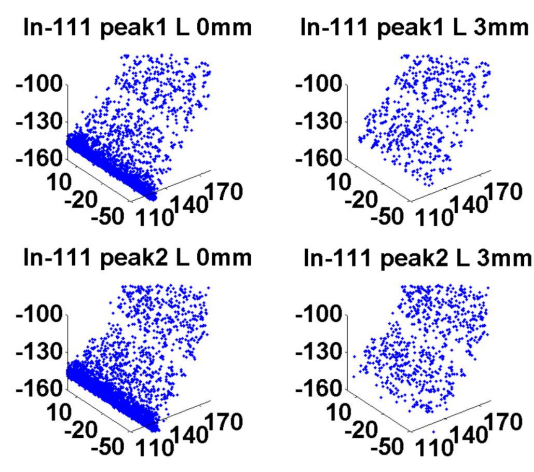

In-111 peak2 $L 3 \mathrm{~mm}$
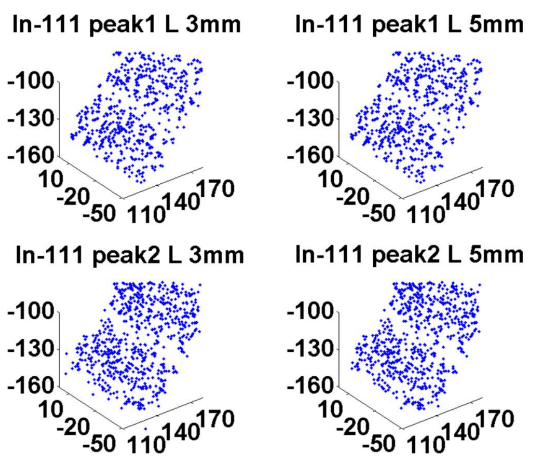

Fig. 12. Distribution of counts along the last detector of the partial ring obtained with ${ }^{111}$ In for variable L shield.

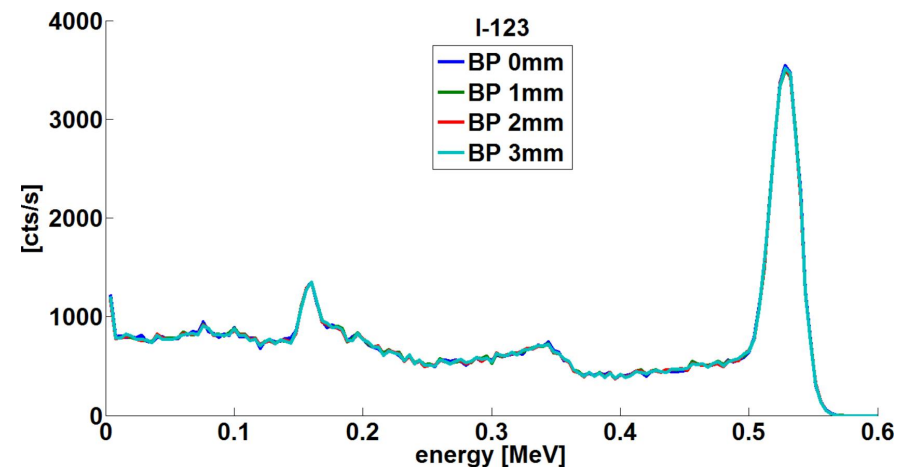

Fig. 13. Energy spectra obtained with ${ }^{123} \mathrm{I}$ for variable BP shields.

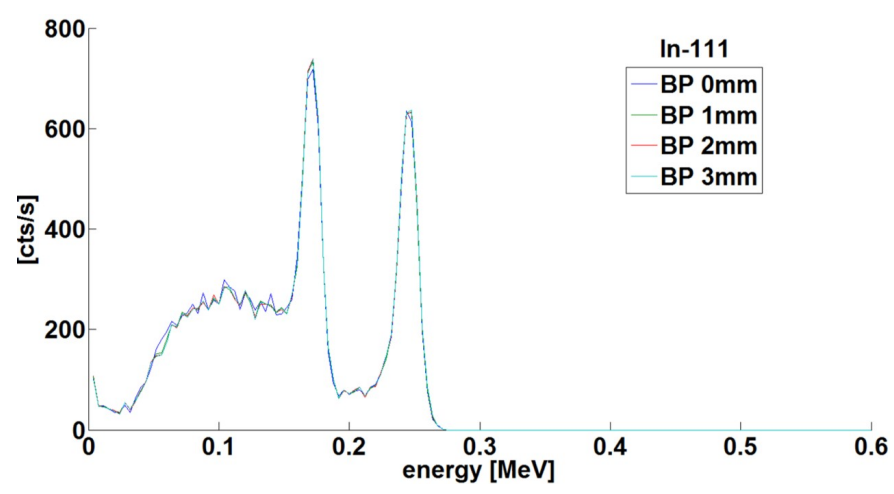

Fig. 14. Energy spectra obtained with ${ }^{111}$ In for variable BP shields.

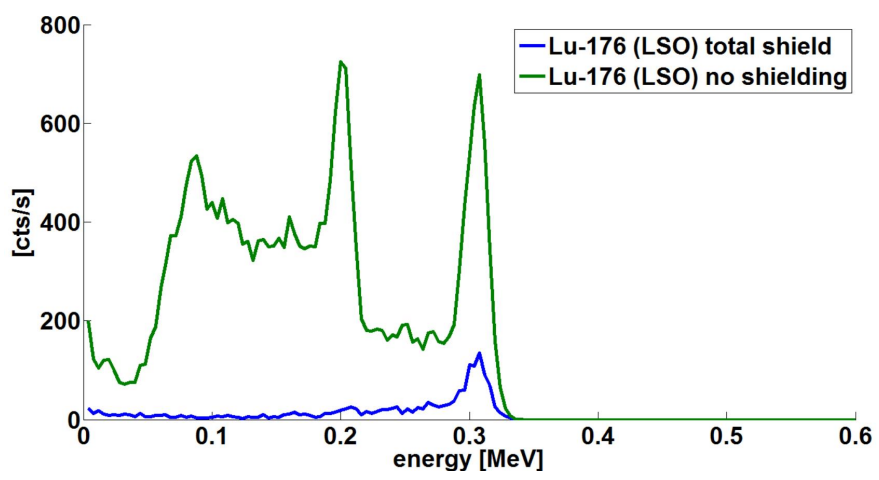

Fig. 15. Energy spectra obtained for ${ }^{176} \mathrm{Lu}$ with and without shielding.

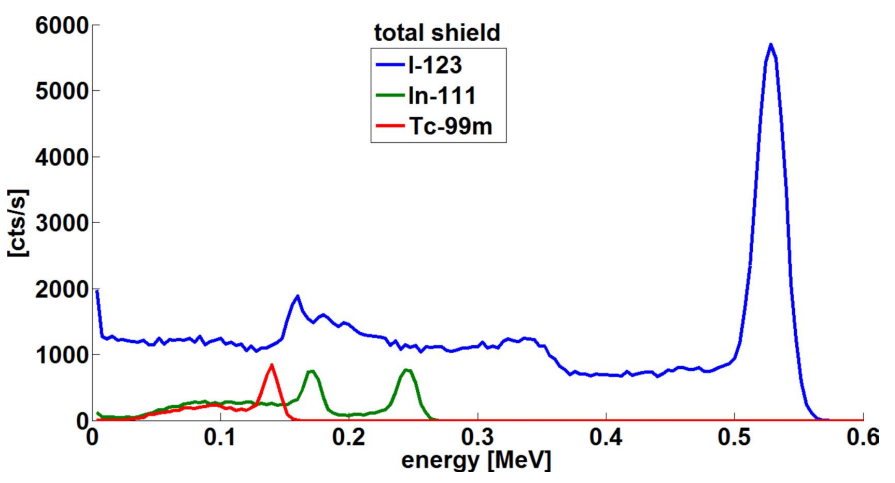

Fig. 16. Energy spectra obtained for ${ }^{99 \mathrm{~m}} \mathrm{Tc},{ }^{111} \mathrm{In}$ and ${ }^{123} \mathrm{I}$ with the final shielding.

For the B shielding, a thickness of $2 \mathrm{~mm}$ reduces the accumulation of ${ }^{123} \mathrm{I}$ counts at the back of the detector (Fig. 9). However, in the energy spectrum, the peak at $159 \mathrm{keV}$ is broadened by scatter from the high energy peak (Fig. 5), making $t_{\mathrm{B}}=3 \mathrm{~mm}$ a better option. This shielding thickness is also suitable in the case of ${ }^{111}$ In (Fig. 6 and Fig. 10).

When comparing the simulations with variable $\mathrm{L}$ shield thickness, the energy spectra are not affected by the thickness, so it is important to check the count-distribution. The edge effects in the last detectors of the partial ring disappear with $t_{\mathrm{L}}=5 \mathrm{~mm}$ for both ${ }^{123} \mathrm{I}$ and ${ }^{111} \mathrm{In}$ (Fig. 11 and Fig. 12).

We observed no differences in the energy spectra obtained for different thicknesses of the base plates (Fig. 13 and Fig. 14), so the best option for support should be considered.

Based on these results, the suggested shielding for the SPECT insert is $t_{\mathrm{F}}=6 \mathrm{~mm}, t_{\mathrm{E}}=2 \mathrm{~mm}, t_{\mathrm{B}}=3 \mathrm{~mm}$, and $t_{\mathrm{L}}=5 \mathrm{~mm}$. With this shielding, the photopeak count rate per detector reduces by a factor of 94 for ${ }^{111} \mathrm{In}(171 \mathrm{keV})$ and 39 for ${ }^{123} \mathrm{I}(159 \mathrm{keV})$. The activity in the FOV is representative of the activity in the brain, but not outside the FOV, where it will accumulate only in some organs of the patient. Therefore, the high contribution from the high energy photons in the case of ${ }^{123} \mathrm{I}$ is likely to be overestimated compared to practice, due to the use of the NEMA phantom in the simulations. The estimated weight of the total shielding is $5.4 \mathrm{~kg}$, which is not a limitation for the system.

Regarding the effects of the PET LSO crystals, without shielding, there is a relatively high contribution of ${ }^{176} \mathrm{Lu}$ photons in the range of $100-200 \mathrm{keV}$. When simulating with the total shielding selected previously, the effect of the LSO intrinsic activity is negligible for the energies of interest (Fig. 15).

As future work, we plan to confirm our results with a more realistic phantom.

\section{Conclusion}

Gamma shielding of the SPECT detectors plays an important role in image quality, specially for installation 
in a PET/MRI system due to the intrinsic activity of the PET LSO crystals.

This work suggests that a $\mathrm{Pb}$ shielding configuration with thickness of $6 \mathrm{~mm}$-front, $2 \mathrm{~mm}$-end, $3 \mathrm{~mm}$-back, and $5 \mathrm{~mm}$-lateral is appropriate for the SPECT insert.

\section{REFERENCES}

[1] G. Delso, S. Furst, B. Jakoby, R. Ladebeck, C. Ganter, S. Nekolla, M. Schwaiger, and S. Ziegler, "Performance Measurements of the Siemens mMR Integrated Whole-Body PET/MR Scanner," Journal of Nuclear Medicine, vol. 52, pp. 1-9, 2011.

[2] D. Salvado, K. Erlandsson, A. Bousse, P. van Mullekom, and B. Hutton, "Novel Collimation for Simultaneous SPECT/MRI," IEEE Nuclear Science Symposium and Medical Imaging Conference, 2014.

[3] P. Busca, C. Fiorini, A. Butt, M. Occhipinti, R. Quaglia, P. Trigilio, G. Nemeth, P. Major, T. Bukki, K. Nagy, C. Piemonte, A. Ferri, A. Gola, J. Rieger, and T. Niendorf, "Development of a highresolution detection module for the INSERT SPECT/MRI system," EJNMMI Physics, 2014.

[4] O. Ott, "Photon Emission Probabilities of $176 \mathrm{Lu}$," International Journal of Radiation Applications and Instrumentation Part A, Applied Radiation and Isotopes, vol. 70, no. 9, pp. 1886-92, 2012.

[5] R. Yao, T. Ma, and Y. Shao, "Lutetium oxyorthosilicate (LSO) intrinsic activity correction and minimal detectable target activity study for spect imaging with a LSO-based animal PET scanner," Physics in Medicine and Biology, vol. 53, pp. 4399-415, 2008.

[6] S. Jan, G. Santin, D. Strul, S. Staelens, K. Assie, D. Autret, S. Avner, V. Breton, A. F. Chatziioannou, Y. Chung, S. Glick, C. Groiselle, S. Kerhoas-Cavata, V. Kohli, M. Krieguer, D. V. der Laan, F. Lamare, C. Lartizien, M. Maas, F. Mayet, F. Melot, C. Merheb, E. Pennacchio, M. Rey, C. Schmidtlein, L. Simon, T. Song, J. Vieira, D. Visvikis, R. V. de Walle, E. Wieers, R. Barbier, M. Bardies, P. Bloomfield, D. Brasse, P. Bruyndonckx, I. Buvat, Y. Choi, C. Comtat, D. Donnarieix, L. Ferrer, D. Guez, P. Honore, A. Kirov, M. Koole, G. Largeron, D. Lazaro, L. Maigne, J. Perez, U. Pietrzyk, F. Rannou, D. Schaart, and C. Morel, "GATE: a simulation toolkit for PET and SPECT," Physics in Medicine and Biology, vol. 49, pp. 4543-61, 2004. 